\title{
EOESGC: Predicting miRNA-disease Associations Based on Embedding of Embedding and Simplified Graph Convolutional Network
}

\section{Shanchen Pang}

China University of Petroleum

\section{yu Zhuang}

China University of Petroleum

Xinzeng Wang ( $\nabla$ wangelxz@126.com )

Shandong University of Science and Technology

\section{Fuyu Wang}

China University of Petroleum

\section{Sibo Qiao}

China University of Petroleum

\section{Research Article}

Keywords: miRNAdisease associations, Embedding of Embedding, Simplified Graph Convolutional Network, Coupled Heterogeneous Graph

Posted Date: August 26th, 2021

DOI: https://doi.org/10.21203/rs.3.rs-831662/v1

License: (c) (1) This work is licensed under a Creative Commons Attribution 4.0 International License. Read Full License 


\title{
RESEARCH
}

\section{EOESGC: Predicting miRNA-disease associations based on Embedding of Embedding and Simplified Graph Convolutional Network}

\author{
Shanchen Pang ${ }^{1}$, Yu Zhuang ${ }^{1}$, Xinzeng Wang ${ }^{2 *}$, Fuyu Wang ${ }^{1}$ and Sibo Qiao ${ }^{1}$
}

\footnotetext{
${ }^{*}$ Correspondence:

wangelxz@126.com

${ }^{1}$ College of Computer Science and

Technology, China University of

Petroleum, Qingdao, China

Full list of author information is

available at the end of the article
}

\begin{abstract}
Background: A large number of biological studies have shown that miRNAs are inextricably linked to many complex diseases. Studying the miRNA-disease associations could provide us a root cause understanding on the underlying pathogenesis in which promotes the progress of drug development. However, traditional biological experiments are very time consuming and costly. Therefore, we come up with more efficient models to solve this challenge.
\end{abstract}

Results: In this work, we propose a deep learning model called EOESGC to predict potential miRNA-disease associations based on embedding of embedding and simplified convolutional network. Firstly, a coupled heterogeneous graph is constructed by using the integrated disease similarity, integrated miRNA similarity and miRNA-disease association networks where parts of the connected edges with less similarity values are removed to simplify the graph structure. The initial feature representation of nodes in the graph is learned using the embedding of embedding model(EOE) based on the principle that the nodes with associations are close to each other and the nodes without association are far from each other. The use of EOE can effectively learn the positional information among nodes and protect the graph structure information to some extent. Then the initial features of the nodes are fed into the simplified graph convolutional network(SGC), and in this step we only use miRNA-disease association network to further simplify the graph structure and thus reduce the computational complexity. Finally, feature embeddings of both miRNA and disease spliced into the MLP for prediction. The two graph simplifications of our model effectively reduce the computational difficulty, and the experimental results show that our model can indeed predict the potential miRNA-disease associations effectively. Compared with the latest published models, our model shows better results. On EOESGC evaluation part, the AUC, AUPR and F1 of our model are 0.9658 , 0.8543 and 0.8644 by $5-$ fold cross validation respectively. In addition, we predict the top 20 potential miRNAs for breast cancer and lung cancer, most of which are validated in the dbDEMC and HMDD3.2 databases.

Conclusion: The comprehensive experimental results show that EOESGC can effectively identify the potential miRNA-disease associations.

Keywords: miRNA-disease associations; Embedding of Embedding; Simplified Graph Convolutional Network; Coupled Heterogeneous Graph

\section{Background}

As a kind of non-coding RNA(ncRNA), miRNA was once thought to be the medium of transcriptional noise from RNA to protein [1-4]. However, this idea was 
proved wrong, and it was verified that non-coding RNA plays an important role in various biological effects $[1,5]$. miRNA is endogenous, evolutionarily conserved single stranded ncRNA that regulates gene expression through complementary base pairing with corresponding target RNA(mRNA) sequences [6-8]. More and more studies had shown that miRNA was closely related to the generation of complex diseases, such as various cancers, diabetes, Alzheimer's disease and other diseases [9-13]. In particular, miRNA act as oncogenes or tumor inhibitors in the generation and metastasis of some cancers, including breast cancer [11] and lung cancer [13]. Since biological experiments are very expensive, more and more researchers hope to use some advanced methods to predict potential miRNA-disease associations on the basis of existing data of proved miRNA-disease associations. Most of the methods proposed so far rely on the hypothesis that functional similarity of miRNAs is associated with similar diseases [14]. The following are several methods for predicting miRNA-disease associations based on graph encoders, random walk, machine learning, and graph convolutional neural network.

Nowdays, graph neural networks have shown their superior performance, such as graph autoencoder. Firstly, three models based on variational autoencoders are introduced. Firstly, three models based on variational autoencoders are introduced. Ji et al. [15] proposed a semi-supervised model(SVAEMDA), which was a novel feature learning approach to obtain their feature representations from an integrated set of miRNA and disease similarity network. SVAEMDA used known miRNA-disease associations in the form of cascaded dense vectors to train predictors based on variable auto-encoders. The reconstruction probability of predictors was used to measure the micronucleic miRNA-disease association. In addition, the model did not need to use negative samples to reduce noise data. Zhang et al. [16] also proposed an unsupervised deep learning framework with variable autoencoder to predict miRNA-disease associations by constructing two spliced matrices as autoencoder(VAE) inputs where VAE learned the potential representation of input data, and reconstructed the data from the learned distribution. The association score of miRNA-disease was obtained by using the trained VAE model. Liu et al. [17] proposed a framework based on stacked autoencoder and XGBoost to predict the potential miRNA-disease associations(SMALF). This model differs from the two previous models as it used an autoencoder to extract miRNA and potential feature vectors of disease, rather than acting as a classifier. It used XGBoost to predict positional miRNA-disease associations.

Secondly, motivated by word2vec, a random walk algorithm was used in the graph to obtain the sequence of nodes and thus the embedding representation of the nodes. Numerous studies had confirmed that the use of random walk algorithm can effectively predict miRNA-disease associations. Thus, we introduce three models based on random walk. Niu et al. [18] constructed a prediction model based on random walk and binary regression, which extracted the features of the miRNAs by restarting the random walk and used binary logistic regression to score the new miRNA-disease associations. Yu et al. [19] proposed a three-layer heterogeneous network combined with non-equilibrium random walk for the miRNA-disease association prediction model (TCRWMDA). This model enabled the construction of a three-layer heterogeneous network, which enriched the information in the basic network and enabled the mining of more effective information between the networks. 
Dai et al. [20] proposed a double random walk based on Logistic weighted profile to explore miRNA-disease associations model (LWBRW). The special feature during the process of constructing the this network. Logistic function was used to extract valuable information. Weighted known proximity (WKNKN) was used to preprocess the known association matrix, and the new miRNA-disease association was inferred by double random walk on the miRNA network and the disease network using the LWBRW method.

Thirdly, traditional machine learning methods are simple, but still have good results. The random forest algorithm had also made outstanding contributions in miRNA - disease associations prediction. Here, three models based on random forest are mainly introduced. Chen et al. [21] proposed a random forest-based method to predict the miRNA-disease associations(RFMDA), using feature selection based on positive and negative sample feature frequencies to reduce the dimension of the sample space. A random forest model was trained to obtain an association score between miRNA and disease. Later, Yao et al. [22] proposed an improved RF model(IRFMDA). Different from Chen's multi-attribute decision analysis method, this model utilized the importance score of $\mathrm{RF}$ variables to realize feature selection, which could effectively reduce the influence of redundancy and noise information, and selected more valuable samples to represent samples, thus improving the prediction ability of the model. Zheng et al. [23] proposed a machine learning approach(MLMDA) to predict and verify miRNA-disease associations by integrating heterogeneous information sources. This model used k-mer sparse matrix to extract miRNA sequence information and other similarity information, which then implements an autoencoder to extract the most representative features of these features. In the end, random forest classifiers are deployed to predict miRNA-disease associations.

Finally, graph convolutional neural networks have shown powerful advantages in the processing of complex graphs, which has led to an increasing number of researchers using graph convolutional neural networks to solve problems. Three models based on graph convolutional neural network are introduced. Peng et al. [24] implemented a convolutional neural network-based framework(MDA-CNN) for predicting miRNA-disease associations by combining similarities between piconucleic acids, similarities between diseases, and interactions between proteins. Chu et al. [25] proposed a new graph sampling method by using feature graph and topology graph to identify miRNA-disease association(MDA-GCNFTG) through graph convolution. This method modeled on the basis of the potential association of feature space and the structural relationship of miRNA-disease associations data where this model could predict not only new miRNA-disease associations, but also new disease-related miRNAs under unbalanced sample distribution. Tang et al. [26] proposed a multi-view and multi-channel attention convolutional network to predict the potential miRNA-disease associations(MMGCN). GCN was used to extract miRNA and node features from different similarity views, and the model used node embedding learned from multi-channel attentional enhancement to make association prediction.

In conclusion, each of these proposed models has its own advantages and disadvantages. Based on previous experience, we proposed a model based on embedding 
of embedding and simplified graph convolutional network, which not only can guarantee the positional information between related nodes, but also effectively aggregate the information between neighbors. Thus it effectively improves the ability of miRNA-disease associations prediction.

\section{Methods}

We present a novel framework for predicting the potential miRNA-disease associations. As shown in Figure 1, the framework consists of four steps in total:

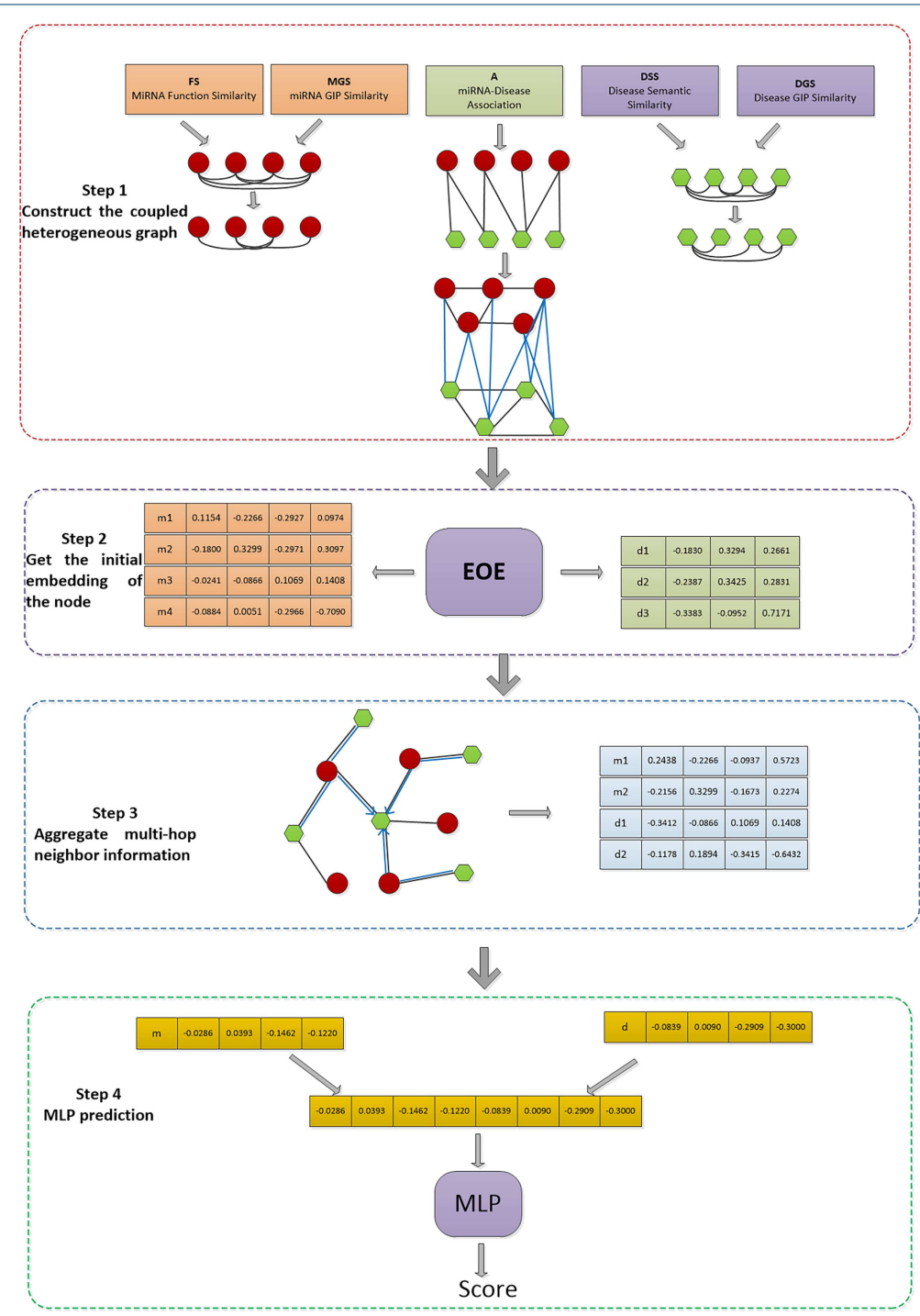

Figure 1 Flow chart of EOESGC

- The first step is to construct the coupled heterogeneous graph, where we use the disease similarity, miRNA similarity and confirmed miRNA-disease association networks to construct the graph and remove the edges with less similarity to reduce the complexity of the graph.

- The second step uses the link-based node embedding model-EOE to obtain the embedding representation of the nodes. The rules of this model are nodes 
with links are close to each other, while nodes without links are far from each other. Based on these two rules, EOE can learn the positional information between nodes effectively.

- The third step is node feature propagation through SGC, which results in the final low-dimensional embedding of the nodes. Each layer of convolution of this model can aggregate multi-hop neighbor information, so more neighbor information can be aggregated with fewer convolution layers. SGC can effectively learn the topological features of the graph.

- The last step is to feed the final embedded splicing into the MLP for prediction.

\section{Database}

A coupled heterogeneous graph consists of two distinct but related sub-nets connected by inter-network edges [27]. Consists of two distinct but related sub-nets connected by inter-network edges. The term "different" implies that the vertices of the two sub-networks are of different node types. The term "correlation" implies that the vertices of two sub-networks have a particular interaction. To construct a miRNA-disease couplied heterogeneity graph, we downloaded data from the HMDD2.0 database [28] containing 495 miRNAs, 383 diseases, and 5430 confirmed miRNA-disease associations. We use the adjacency matrix A to represent miRNA-disease associations where $A_{i j}=1$ means there is a interaction between miRNA i and disease $\mathrm{j}$, while $A_{i j}=0$ means there is no relationship. In the experiment stage, we used dbDEMC [29] and HMDD3.2 databases as the verification database to verify the accuracy of the EOESGC model we proposed.

Disease similarity network

We effectively combine disease semantic similarity with disease Gaussian interaction profile kernel similarity to construct disease similarity network. In order to ensures edges among disesase nodes are valid, we set a threshold and remove the link below the threshold. Therefore, the disease similarity is calculated as follows:

$$
D S^{\prime}\left(d_{i}, d_{j}\right)=\alpha \frac{S S^{1}\left(d_{i}, d_{j}\right)+S S^{2}\left(d_{i}, d_{j}\right)}{2}+(1-\alpha) G S\left(d_{i}, d_{j}\right)
$$

The first semantic similarity is $S S^{1}$, the second semantic similarity is $S S^{2}$, and the Gaussian interaction profile kernel similarity is GS. In the experiment, $\alpha$ represents a scaling factor. The disease similarity obtained after removing data with low similarity according to the threshold h:

$$
D S\left(d_{i}, d_{j}\right)=\left\{\begin{array}{cl}
D S^{\prime}\left(d_{i}, d_{j}\right) & D S^{\prime}\left(d_{i}, d_{j}\right) \geq h \\
0 & \text { other else }
\end{array}\right.
$$

Disease semantic similarity model 1

Medical Subject Headings(MESH) [30] is the authoritative Subject list compiled by the United States National Library of Medicine. It is a normalized and expandable dynamic thesaurus. Mesh is a collection of more than 18,000 medical topics that we use to study the relationships between diseases. Disease can be described as 
a Directed Acyclic Graph $\left(\mathrm{DAG}=N_{d}, E_{d}\right)$, where $N_{d}$ is the node set of d and it's ancestor nodes, $E_{d}$ is edge set [31]. As Figure 2 shows the DAG of two diseases.

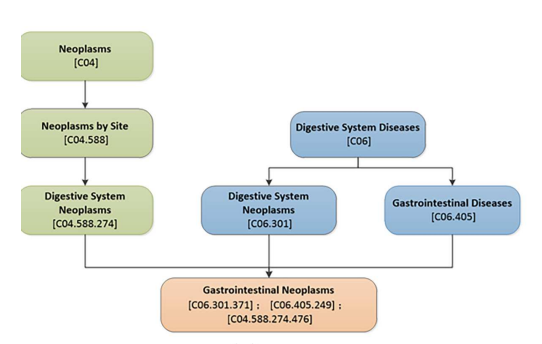

(a)

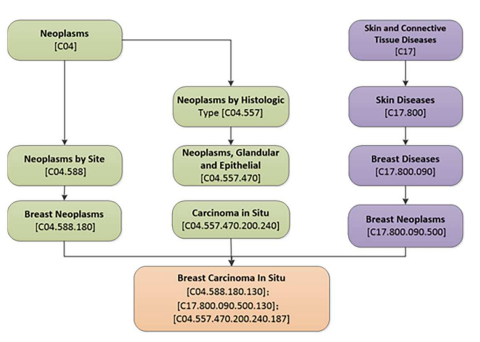

(b)

Figure 2 directed acyclic graph of diseases. (a) directed acyclic graph of breast cancer in situ, (b) directed acyclic graph of gastrointestinal neoplasms

In order to calculate the similarity of two disease semantics based on DAG(D), we need to calculate the semantic contribution score for each disease in the graph. We define the contribution score of disease $d$ to disease $D$ in $D A G(D)$ as:

$$
D_{D}^{1}=\left\{\begin{array}{ccc}
1 & & d=D \\
\max \left\{\Delta * D_{D}^{1}\left(d^{\prime}\right) \mid d^{\prime} \in\right. \text { the } & \text { children } \quad \text { of } \quad d\} & d \neq D
\end{array}\right.
$$

where $\Delta=0.5$ is a decay factor indicating that the more distant nodes from disease $\mathrm{D}$ contribute less to the semantics of disease D. The semantic value of disease D is calculated based on the semantic contribution score of the disease nodes in DAG(D).

$$
D V^{1}(D)=\sum_{d \in N(D)} D_{D}^{1}(d)
$$

If DAG (A) and DAG (B) have same diseases, we consider disease A and disease B to be similar. Therefore, the first semantic similarity between two diseases is defined as:

$$
S S^{1}=\frac{\sum_{t \in N\left(d_{i}\right) \cap N\left(d_{j}\right)}\left(D_{d_{i}}^{1}(t)+D_{d_{j}}^{1}(t)\right)}{D V^{1}\left(d_{i}\right)+D V^{1}\left(d_{j}\right)}
$$

\section{Disease semantic similarity model 2}

Xuan et al. [32] defined the essential difference between the second disease semantic similarity and the first disease semantic similarity which differs in the calculation of semantic contribution of disease nodes. The ancestor nodes of disease D have $\mathrm{d} 1$ and $\mathrm{d} 2$, and if $\mathrm{d} 1$ appears less frequently in DAG than $\mathrm{d} 2$, then we believe that $\mathrm{d} 1$ has a greater semantic contribution to disease D. Therefore, the semantic contribution score of disease node $\mathrm{d}$ to disease $\mathrm{D}$ is defined as:

$$
D_{D}^{2}(d)=-\log \left(\frac{\text { the number of } D A G_{s} \text { of }}{\text { the number of disease }}\right)
$$


As in model 1, the semantic value of each disease and the semantic similarity of the two diseases are defined as :

$$
\begin{aligned}
& D V^{2}(D)=\sum_{d \in N(D)} D_{D}^{2}(d) \\
& S S^{2}=\frac{\sum_{t \in N\left(d_{i}\right) \cap N\left(d_{j}\right)}\left(D_{d_{i}}^{2}(t)+D_{d_{j}}^{2}(t)\right)}{D V^{2}\left(d_{i}\right)+D V^{2}\left(d_{j}\right)}
\end{aligned}
$$

Disease Gaussian interaction profile kernel similarity (GIP)

Since not all the diseases can be found in the MESH, we use the disease Gaussian interaction profile kernel similarity as a supplement. GIP similarity is calculated for miRNA and disease respectively using the method proposed by Zhao et al. [33]. The adjacency matrix $A \in R^{m * n}$ of miRNA-disease, where each column is used to represent a disease, is defined as $\operatorname{IP}(\mathrm{D})$, where each column is defined as $\operatorname{IP}(\mathrm{D})$ to represent a disease. Then, the Gaussian interaction kernel similarity between diseases $d_{i}$ and $d_{j}$ is defined as:

$$
K D\left(d_{i}, d_{j}\right)=\exp \left(-\gamma_{d}\left\|I P\left(d_{i}\right)-I P\left(d_{j}\right)\right\|^{2}\right)
$$

Where $\gamma_{d}$ is used to control kernel bandwidth, $\gamma_{d}^{\prime}$ is usually set to 0.5 for controlling the kernel bandwidth $\gamma_{d}$ is defined as:

$$
\gamma_{d}=\gamma_{d}^{\prime} / \frac{1}{n} \sum_{i=1}^{n}\left\|I P\left(d_{i}\right)\right\|^{2}
$$

miRNA similarity network

We use miRNA functional similarity and Gaussian interaction profile kernel similarity to construct miRNA similarity network. The Gaussian interaction profile kernel similarity is the same as in the previous section. miRNA similarity is defined as:

$$
M S^{\prime}\left(m_{1}, m_{2}\right)=\alpha F S\left(m_{1}, m_{2}\right)+(1-\alpha) G S\left(m_{1}, m_{2}\right)
$$

where $\alpha$ is the scale factor, FS is miRNA function similarity. We set a threshold value of $\mathrm{h}$, in believing there is no association between miRNAs with a similarity less than $\mathrm{h}$. Therefore, the final miRNA similarity network is defined as:

$$
M S\left(m_{1}, m_{2}\right)=\left\{\begin{array}{cl}
M S^{\prime}\left(m_{1}, m_{2}\right) & M S^{\prime}\left(m_{1}, m_{2}\right) \geq h \\
0 & \text { other else }
\end{array}\right.
$$

According to Wang et al. [31] study, miRNAs with similar functions are often associated with diseases with similar semantics, and the relationship between different diseases can be represented by a Directed Acyclic Graph(DAG) structure. The functional similarity of miRNA is inferred by measuring the similarity of DAG of related diseases. Firstly, the similarity of disease $d_{t}$ to the disease set DT is defined as:

$$
s\left(d_{t}, D T\right)=\max _{1 \leq i \leq k} S\left(d_{t}, d_{i}\right)
$$


If the disease set associated with $m_{1}$ is $D T_{1}$ and the disease set associated with $m_{2}$ is $D T_{2}$, then the functional similarity between and is defined as:

$$
M S\left(m_{1}, m_{2}\right)=\frac{\sum_{1 \leq i \leq m} S\left(d_{i}, D T_{2}\right)+\sum_{1 \leq j \leq n} S\left(d_{j}, D T_{1}\right)}{m+n}
$$

Where $d_{i}$ belongs to $D T_{1}, d_{j}$ to $D T_{2}, \mathrm{~m}$ is the number of diseases contained in $D T_{1}$, and $\mathrm{n}$ is the number of diseases contained in $D T_{2}$.

\section{EOESGC Model}

We combine two embedding models to obtain the embedding of nodes. The first is the link-based graph embedding model-Embedding of Embedding model, which proposed a new graph type called coupled heterogeneous graph, and miRNA-disease network essentially belongs to this type. The EOE model emphasizes that linked vertices should be close to each other and unlinked vertices should be far away from each other. The latter rule is also important. Therefore, the model set different loss functions to satisfy this rule. A harmony matrix $\mathrm{M}$ was proposed to calculate the proximity between different types of nodes. The link-based embedding model can learn the structural characteristics of graph well, and this method is effective and easy to implement. Then, we input the obtained embedding and miRNA-disease association network into the simplified graph convolution network to continue learning node features. The nonlinear GCN [34] is transformed into a simple linear model SGC, which reduces the additional complexity of the GCN by repeatedly eliminating the non-linearity between the GCN layers and folding the resulting function into a linear transformation. This simplified linear SGC model is more efficient on many tasks than GCN and some other GNN networks along with fewer parameters as well. And the embedded model based on convolution can effectively obtain the neighbor information of the node. The EOESGC model does not join the embedding of the two models, but puts the embedding obtained from one model into the second model for training. The experiment proves that this method can effectively learn node embedding.

\section{Embedding of Embedding}

The EOE uses proximity to measure whether there are links between nodes. The larger the degree of proximity is the more similar between two same types of nodes will be, and correlations two different types of nodes will show. We input the similarity matrix of nodes as the original feature. So we define the proximity between two nodes of the same type as follows:

$$
\begin{aligned}
& p\left(d_{i}, d_{j}\right)=\frac{1}{1+\exp \left(-d_{i}^{T} d_{j}\right)} \\
& p\left(m_{i}, m_{j}\right)=\frac{1}{1+\exp \left(-m_{i}^{T} m_{j}\right)}
\end{aligned}
$$

Where $d_{i}$ represents row $\mathrm{i}$ of the disease similarity matrix, $d_{j}$ represents row $\mathrm{j}$ of the disease similarity matrix, $m_{i}$ represents row i of the miRNA similarity matrix, $m_{j}$ represents row $\mathrm{j}$ of the miRNA similarity matrix. 
For different types of nodes, the feature matrix $M \in R^{m * n}$ is introduced during the calculation of proximity since their features cannot be directly computed in different feature spaces. Thus the proximity between pairs of nodes of different types is defined as:

$$
p\left(d_{i}, m_{j}\right)=\frac{1}{1+\exp \left(-d_{i}^{T} M m_{j}\right)}
$$

In order to satisfy that bounded nodes with small probability and boundless vertices with large probability should receive greater penalties. The loss function is defined as:

$$
\begin{aligned}
\operatorname{loss}= & -\left[\sum_{\left(d_{i}, d_{j}\right) \in E_{d}}\left(W_{d}\right)_{i j} \log \left(p\left(d_{i}, d_{j}\right)\right)\right. \\
& +\sum_{\left(m_{i}, m_{j}\right) \in E_{m}}\left(W_{m}\right)_{i j} \log \left(p\left(m_{i}, m_{j}\right)\right) \\
& \left.+\sum_{\left(d_{i}, m_{j}\right) \in E_{d m}}\left(W_{d m}\right)_{i j} \log \left(p\left(m_{i}, d_{j}\right)\right)\right] \\
& -\left[\sum_{\left(d_{i}, d_{j}\right) \notin E_{d}} \log \left(1-p\left(d_{i}, d_{j}\right)\right)\right. \\
& +\sum_{\left(m_{i}, m_{j}\right) \notin E_{m}} \log \left(1-p\left(m_{i}, m_{j}\right)\right) \\
& \left.+\sum_{\left(d_{i}, m_{j}\right) \notin E_{d m}} \log \left(1-p\left(d_{i}, m_{j}\right)\right)\right]
\end{aligned}
$$

Where $E_{d}$ is the set of edges between diseases, $E_{m}$ is the set of edges between miRNAs, $E_{d m}$ is the edge set between disease and miRNA, $W_{d}$ is the weight between disease, $W_{m}$ is the weight between miRNA, and $W_{d m}$ is the weight between disease and miRNA.

\section{Simplifying Graph Convolutional Network}

In the traditional GCN, each layer can only aggregate the information of directly connected neighbors. while in SGC, we can set the information aggregation of $\mathrm{K}$-hop neighbors at each layer. SGC consists of two parts, a fixed feature extractor and a linear logistic regression classifier. In our proposed framework, only the feature extractor is used to obtain the embedded representation of nodes. Because miRNA and disease embedding learned from EOE model still belong to two different feature spaces, they are first mapped to the same feature space.

We map diseases and miRNAs into the $\mathrm{Z}$ dimensional feature space as follows:

$$
\begin{aligned}
& X_{m}=W^{M} \cdot x_{m} \\
& X_{d}=W^{D} \cdot x_{d}
\end{aligned}
$$

Where $x_{m}$ and $x_{d}$ are miRNA embedding and disease embedding output by EOE, $W^{M}, W^{D} \in R^{Z}$ are the mapping matrices. Then, the feature embedding of the 
disease and miRNA are fed into the SGC. The convolution operation for each layer as follows:

$$
\begin{aligned}
& \tilde{A}=A+I \\
& S=\tilde{D}^{-\frac{1}{2}} \tilde{A} \tilde{D}^{-\frac{1}{2}} \\
& S^{k}=S \cdots S S \\
& \bar{X}=S^{k} X
\end{aligned}
$$

Where $\mathrm{A}$ is the adjacency matrix of the graph, I is the identity matrix; D is the degree matrix of $\mathrm{A}, \mathrm{K}$ is the step size.

Finally, the output disease embedding and miRNA embedding are spliced, and make predictions with MLP. This step uses the cross-entropy loss function to optimize the model.

$$
\operatorname{loss}=-[y \log \tilde{y}+(1-y) \log (1-\tilde{y})]
$$

Where $\mathrm{y}$ is the edge label, $\tilde{y}$ is the predict score.

\section{Results}

Comparisons with the state-of-the-art methods

In order to prove the superiority of the proposed model, we compare it with several more excellent models recently proposed. During the experiment, the same data set is used and the AUC values of each model were obtained by using 5 -fold cross validation for comparison. Models LWPCMF [35], VAGMF [36], SMALF [17], CEMDA [37] and ICFMDA [38] are compared. As shown in Table 1, our proposed model is significantly better than the latest model.

Table 1 The ROC curves of EOESGC and baseline

\begin{tabular}{cc}
\hline Method & AUC \\
\hline EOESGC & 0.9658 \\
LWPCMF & 0.9411 \\
VAFMF & 0.9280 \\
SMALF & 0.9503 \\
CEMDA & 0.9203 \\
ICFMDA & 0.9045 \\
\hline
\end{tabular}

\section{Experimental approaches and evaluation criteria}

To verify the validity of our proposed EOESGC model, we conduct experiments on the HMDD2.0 database and evaluate the model ferformance by using 5 -fold cross validation and 10 -fold cross validation. Considering the large difference in the number of positive and negative samples during the experiment, we randomly select 5 negative samples for each positive sample to form the experimental data, thus achieving the function of balancing the data set. The results are shown in Figure 3. The AUC of our model for 5 -fold cross validation is 0.9658 and the AUPR is 0.8543 , the AUC for 10 -fold cross validation is 0.9644 and the AUPR is 0.8540 . 


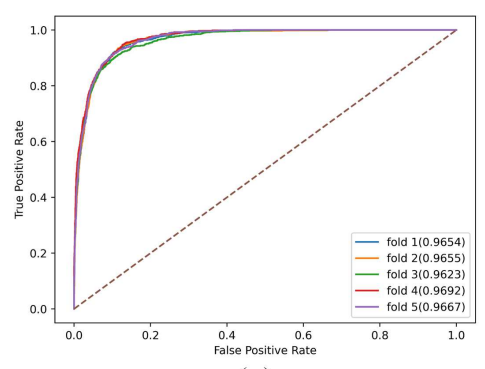

(a)

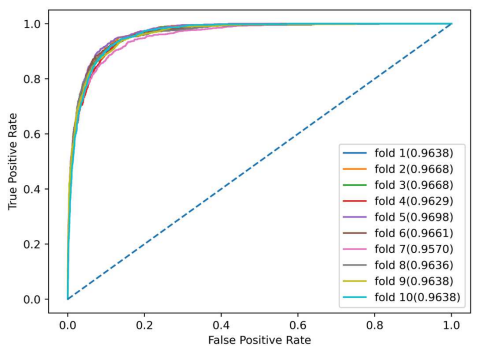

(c)

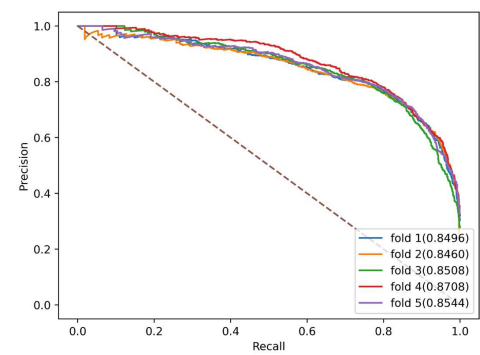

(b)

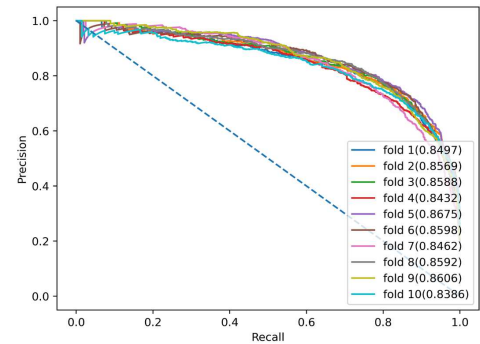

(d)

Figure 3 Cross validation results. (a): 5 -fold cross validated ROC curve with a mean AUC of 0.9658; (b): 5-fold cross validated PR curve with a mean AUPR of 0.8543; (c): 10-fold cross validated ROC curve with a mean AUC of 0.9644 ; (d): 10 -fold cross validated PR curve with a mean AUPR of 0.8540

\section{Parameter sensitivity analysis}

Different embedding dimensions will cause different model training speed and cost. In order to select the optimal embedding dimension, we conduct a 5-fold cross validation experiment with different dimensions. The experimental results are shown in Figure 4. When the embedding dimension is not greater than 64, the value of AUC shows an increasing trend, but when the dimension is greater than 64 , the value of AUC starts to decrease. Therefore, we choose 64 as the embedding dimension of the node.

\section{Compare the different combination types}

Network representation learning has been widely used in the association prediction task of miRNA-disease. Some of the common methods include SDNE [39], Node2vec [40], Deepwalk [41], and LINE [42]. And in order to better gather neighbor information and learn the topological characteristics of graphs, researchers continuously improve the convolution operation and propose different convolution layers for us to use. In this paper, we use SGC model, so that each layer of convolution operation can gather more distant neighbor nodes, and achieve better results in our prediction task. At the same time, we also used GCN, GraphSage [43] and TAG [44] models as our comparison models. Therefore, a total of 16 different models can be formed by 4 kinds of embedded models and 4 kinds of graph convolution. In order to eliminate the contingency of the experimental results, we repeated the 5 -fold cross validation for three times, and took the average value as the final result. The experimental results of these combined models are shown in Table 2. 


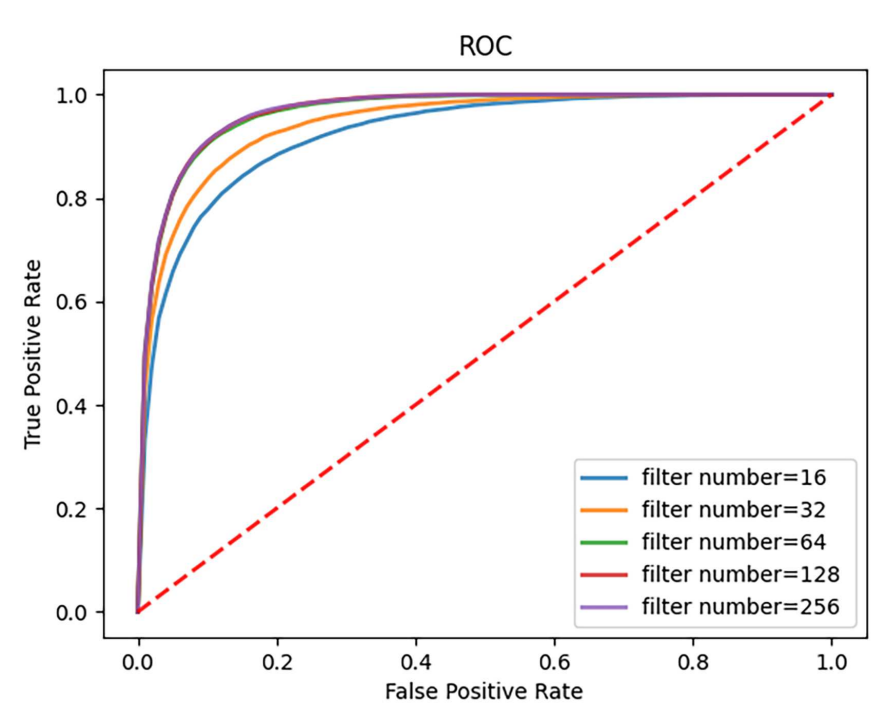

Figure 4 Parameter analysis of EOESGC

Table 2 The different combination types result

\begin{tabular}{cccc}
\hline Model & AUC & AUPR & F1-score \\
\hline EOESGC & 0.9658 & 0.8543 & 0.8644 \\
EOEGCN & 0.9178 & 0.7193 & 0.7973 \\
EOEGraphSAGE & 0.9301 & 0.7685 & 0.8169 \\
EOETAG & 0.9501 & 0.8147 & 0.8419 \\
SDNESGC & 0.9345 & 0.7952 & 0.8237 \\
SDNEGCN & 0.9027 & 0.6681 & 0.7707 \\
SDNEGraphSAGE & 0.9547 & 0.8342 & 0.8481 \\
SDNETAG & 0.9482 & 0.8137 & 0.8406 \\
LINESGC & 0.9206 & 0.7239 & 0.7991 \\
LINEGCN & 0.8839 & 0.5925 & 0.7472 \\
LINEGraphSAGE & 0.9298 & 0.7617 & 0.8123 \\
LINETAG & 0.9279 & 0.7455 & 0.8045 \\
DeepWalkSGC & 0.9185 & 0.7215 & 0.7937 \\
DeepWalkGCN & 0.8792 & 0.6004 & 0.7520 \\
DeepWalkGraphSAGE & 0.9119 & 0.6972 & 0.7839 \\
DeepWalkTAG & 0.9211 & 0.7262 & 0.7957 \\
Node2vecSGC & 0.7569 & 0.4735 & 0.5301 \\
Node2vecGCN & 0.4655 & 0.1494 & 0.4545 \\
Node2vecGraphSAGE & 0.7030 & 0.2396 & 0.4545 \\
Node2vecTAG & 0.7922 & 0.4228 & 0.4585 \\
\hline
\end{tabular}

\section{Case Study}

Breast neoplasms are common cancers that threaten women's health worldwide and are also one of the leading causes of nausea in women's deaths [45].In recent years, gene diagnosis and gene therapy of breast cancer have become a hot topic.Studies have shown that miRNA, as a regulatory factor, plays an important role. For example, low expression of mir-195 can be easily observed in breast cancer cell lines and tissue samples from chemotherapy-sensitive or drug-resistant patients [45]. In addition, mir-195 can decrease the survival rate and increase apoptosis of breast tumor cells by down-regulating the expression of Raf-1, Bcl-2 and $\mathrm{P}$-glycoprotein [45]. Therefore, it is necessary to use advanced methods to predict the potential miRNA related to breast neoplasms, so we predicted the top 20 miRNAs associated with it, as shown in Table 3. 
Table 3 The top 20 potential miRNAs related to Breast Neoplasms

\begin{tabular}{cc|cc}
\hline miRNA & Evidence & miRNA & Evidence \\
\hline hsa-mir-142 & dbDEMC,HMDD3.2 & hsa-mir-106a & dbDEMC,HMDD3.2 \\
hsa-mir-150 & dbDEMC,HMDD3.2 & hsa-mir-574 & dbDEMC,HMDD3.2 \\
hsa-mir-181c & dbDEMC,HMDD3.2 & hsa-mir-15b & dbDEMC,HMDD3.2 \\
hsa-mir-192 & dbDEMC,HMDD3.2 & hsa-mir-30e & dbDEMC,HMDD3.2 \\
hsa-mir-494 & dbDEMC,HMDD3.2 & hsa-mir-138 & dbDEMC,HMDD3.2 \\
hsa-mir-378a & dbDEMC,HMDD3.2 & hsa-mir-424 & dbDEMC,HMDD3.2 \\
hsa-mir-184 & dbDEMC,HMDD3.2 & hsa-mir-372 & dbDEMC,HMDD3.2 \\
hsa-mir-208b & dbDEMC & hsa-mir-212 & dbDEMC,HMDD3.2 \\
hsa-mir-208a & dbDEMC,HMDD3.2 & hsa-mir-134 & dbDEMC,HMDD3.2 \\
hsa-mir-99a & dbDEMC,HMDD3.2 & hsa-mir-28 & dbDEMC \\
\hline
\end{tabular}

Lung neoplasms are the most common type of nausea and has a high mortality rate. Previous studies have shown that miRNA is involved in almost every process of lung cancer, including tumor progression, angiogenesis, invasion and metastasis. For example, the expression level of $\mathrm{miR}-29 \mathrm{~s}$ was found to be inversely correlated with DNA methyltransferase 3A (DNMT3A) and DNA methyltransferase 3B (DNMT3B) in lung cancer tissues by controlling methylation to inhibit the reexpression of tumor suppressor genes and inhibit tumogenesis [46]. We still used our proposed framework to predict the first 20 miRNAs associated with lung cancer, as shown in Table 4.

Table 4 The top 20 potential miRNAs related to Lung Neoplasms

\begin{tabular}{cc|cc}
\hline miRNA & Evidence & miRNA & Evidence \\
\hline hsa-mir-16 & dbDEMC,HMDD3.2 & hsa-mir-378a & dbDEMC \\
hsa-mir-122 & dbDEMC,HMDD3.2 & hsa-mir-20b & dbDEMC \\
hsa-mir-15a & dbDEMC,HMDD3.2 & hsa-mir-23b & dbDEMC \\
hsa-mir-106b & dbDEMC,HMDD3.2 & hsa-mir-184 & dbDEMC \\
hsa-mir-195 & dbDEMC,HMDD3.2 & hsa-mir-342 & dbDEMC,HMDD3.2 \\
hsa-mir-429 & dbDEMC & hsa-mir-208a & HMDD3.2 \\
hsa-mir-373 & dbDEMC,HMDD3.2 & hsa-mir-99a & dbDEMC,HMDD3.2 \\
hsa-mir-451a & dbDEMC,HMDD3.2 & hsa-mir-302b & dbDEMC \\
hsa-mir-141 & dbDEMC,HMDD3.2 & hsa-mir-15b & dbDEMC \\
hsa-mir-302a & dbDEMC & hsa-mir-208b & Unconfirmed \\
\hline
\end{tabular}

\section{Conclusions}

Experiments show that our proposed EOESGC model can be used to predict potential miRNA-disease associations. The coupled heterogeneous graph is constructed by combining miRNA, disease and miRNA-disease association networks. The model combines two feature learning models. First, the embedding representation of the nodes is learned using the EOE, which can effectively learn the positional information of the graph. Then, to reduce the computational effort, we simplify the graph and keep only the associated networks. The node features are learned through SGC. The model can learn the topological information of the graph effectively. The information of the graph can be better learned by using two embedding models. Finally, the MLP is used for prediction. During the experiment, 5 negative samples are randomly selected from each positive sample to form the experimental data in order to balance the uneven data set. The experimental results show that the AUC value of the EOESGC model based on 5-fold cross validation was 0.9658, which is higher than previous methods. The top 20 associated potential miRNA are predicted in lung cancer and breast cancer cases. the validation databases are used dbDEMC and HMDD3.2 databases, and 20, 19 miRNA are identified in the 
validation database. Therefore, the EOESGC model is very effective for predicting potential miRNA-disease associations.

\section{Availability of data and material}

The datasets used and/or analysed during the study is available from the corresponding author on reasonable request. Data can be downloaded from the Human mirorRNA Disease Database:http://www.cuilab.cn/hmdd/

Competing interests

I declare that the authors have no competing interests as defined by BMC, or other interests that might be perceived to influence the results and/or discussion reported in this paper.

Authors' contributions

ZY conceived the prediction method and wrote the paper, PSC, WXZ and QSB modified the paper, and ZY and WFY completed the code implementation. All authors read and approved the final manuscript.

Acknowledgements

Thanks to PSC and WXZ for correcting the paper.

Funding

This work was supported by National Natural Science Foundation of China under grant No.61873281.

Author details

${ }^{1}$ College of Computer Science and Technology, China University of Petroleum, Qingdao, China. ${ }^{2}$ College of Mathematics and Systems Science, Shandong University of Science and Technology, Qingdao, China.

\section{References}

1. Chen, X.: Constructing Incrna functional similarity network based on Incrna-disease associations and disease semantic similarity. Scientific Reports 5, 11338 (2015)

2. Chen, G., Wang, Z., Wang, D., Qiu, C., Liu, M., Xing, C., Zhang, Q., Yan, G., Cui, Q.: Lncrnadisease: a database for long-non-coding rna-associated diseases. Nucleic Acids Research (D1), 983-986 (2013)

3. Ponting, C.P., Oliver, P.L., Reik, W.: Evolution and functions of long noncoding rnas. Cell 136(4), 629-641 (2009)

4. Esteller, M.: Non-coding rnas in human disease. Nat Rev Genet 12(12), 861-874 (2011)

5. Doench, J.G., Peterson, C.P., Sharp, P.A.: The functions of animal micrornas. Nature 7006(431), 350-355 (2004)

6. Rosalind, C., Lee, Rhonda, L.: The c. elegans heterochronic gene lin-4 encodes small rnas with antisense complementarity to lin-14. Cell (1993)

7. Lee RC, A.V.: An extensive class of small rnas in caenorhabditis elegans. Science 5543(294), $862-864$ (2001)

8. Mir, S.M., Rajasekaran, P.: Oncomirs -" micrornas with a role in cancer". American Mathematical Society Contemporary Mathematics, 53-72 (1993)

9. Li, C.F.: Microrna signatures in human cancers. Nature Reviews Cancer 6(11), 857-66 (2006)

10. Huang, Q., Gumireddy, K., Schrier, M., Sage, C.L., Nagel, R., Nair, S., Egan, D.A., Li, A., Huang, G., Klein-Szanto, A.J.: The micrornas mir-373 and mir-520c promote tumour invasion and metastasis. Nature Cell Biology 10(2), 202-210 (2008)

11. Iorio, M. M. V. Ferracin: Microrna gene expression deregulation in human breast cancer. Cancer Research 16(65), 7065-7070 (2005)

12. Latronico, M., Catalucci, D., Condorelli, G.: Emerging role of micrornas in cardiovascular biology. Circulation Research 101(12), 1225-1236 (2007)

13. Yanaihara N, B.E. Caplen N: Unique microrna molecular profiles in lung cancer diagnosis and prognosis. Cancer Research 8(66), 189-198 (2006)

14. Chen, X., Xie, D., Zhao, Q., You, Z.H.: Micrornas and complex diseases: from experimental results to computational models. Briefings in Bioinformatics 20(2), 515-539 (2019)

15. Ji, C., Wang, Y.T., Gao, Z., Li, L., Zheng, C.H.: A semi-supervised learning method for mirna-disease association prediction based on variational autoencoder. IEEE/ACM Transactions on Computational Biology and Bioinformatics PP(99), 1-1 (2021)

16. Zhang, L., Chen, X., Yin, J.: Prediction of potential mirna“"cdisease associations through a novel unsupervised deep learning framework with variational autoencoder. Cells 8(9) (2019)

17. Liu, D., Huang, Y., Nie, W., Zhang, J., Deng, L.: Smalf: mirna-disease associations prediction based on stacked autoencoder and xgboost. BMC Bioinformatics 22(1) (2021)

18. Niu, Y.W., Wang, G.H., Yan, G.Y., Chen, X.: Integrating random walk and binary regression to identify novel mirna-disease association. BMC Bioinformatics 20(1) (2019)

19. Limin, Xianjun, Shen, Duo, Zhong, Jincai, Yang: Three-layer heterogeneous network combined with unbalanced random walk for mirna-disease association prediction. Frontiers in genetics 10, 1316-1316 (2019)

20. Dai, L.Y., Liu, J.X., Zhu, R., Wang, J., Yuan, S.S.: Logistic weighted profile-based bi-random walk for exploring mirna-disease associations. Journal of Computer Science and Technology 36(2), 276-287 (2021)

21. Chen, X., Wang, C.C., Yin, J., You, Z.H.: Novel human mirna-disease association inference based on random forest. Molecular Therapy - Nucleic Acids 13, 568-579 (2018)

22. Yao, D., Zhan, X., Kwoh, C.K.: An improved random forest-based computational model for predicting novel mirna-disease associations. BMC Bioinformatics 20 (2019)

23. Zheng, K., You, Z.H., Wang, L., Zhou, Y., Li, Z.W.: Mlmda: a machine learning approach to predict and validate microrna"cdisease associations by integrating of heterogenous information sources. Journal of Translational Medicine 17(1) (2019) 
24. Jiajie, Peng, Weiwei, Hui, Qianqian, Bolin, Chen, Jianye, Hao, Qinghua: A learning-based framework for mirna-disease association identification using neural networks. Bioinformatics (Oxford, England) (2019)

25. Chu, Y., Wang, X., Dai, Q., Wang, Y., Wei, D.Q.: Mda-gcnftg: identifying mirna-disease associations based on graph convolutional networks via graph sampling through the feature and topology graph. Briefings in Bioinformatics (2021)

26. Tang, X., Luo, J., Shen, C., Lai, Z.: Multi-view multichannel attention graph convolutional network for mirna"cdisease association prediction. Briefings in Bioinformatics (7006) (2021)

27. Xu L, C.J. Wei X: Embedding of embedding (eoe) : Joint embedding for coupled heterogeneous networks. ACM (9), 741-749 (2017)

28. Yang, L., Qiu, C., Tu, J., Geng, B., Yang, J., Jiang, T., Cui, Q.: Hmdd v2.0: a database for experimentally supported human microrna and disease associations. Nucleic Acids Research (D1), 1070 (2014)

29. Zhen, Y., Fei, R., Liu, C., He, S., Gang, S., Qian, G., Lei, Y., Zhang, Y., Miao, R., Ying, C.: dbdemc: a database of differentially expressed mirnas in human cancers. Bmc Genomics 11(Suppl 4), 1-8 (2010)

30. Lipscomb, C.E.: Medical subject headings (mesh). Bulletin of the Medical Library Association 88(3), 265-266 (2000)

31. Cui, Q.: Inferring the human microrna functional similarity and functional network based on microrna-associated diseases. Bioinformatics 26(13), 1644-1650 (2010)

32. Ping, X., Ke, H., Guo, M., Guo, Y., Li, J., Jian, D., Yong, L., Dai, Q., Jin, L., Teng, Z.: Correction: Prediction of micrornas associated with human diseases based on weighted $k$ most similar neighbors. PLOS ONE 8 (2013)

33. Yan, Zhao, Xing, Chen, Jun, Yin: Adaptive boosting-based computational model for predicting potential mirna-disease associations. Bioinformatics (Oxford, England) 1(36), 330-330 (2019)

34. Wu, F., Zhang, T., Souza, A., Fifty, C., Yu, T., Weinberger, K.Q.: Simplifying graph convolutional networks (2019)

35. Meng-Meng, Yin, Zhen, Cui, Ming-Ming, Gao, Jin-Xing, Liu, Ying-Lian: Lwpcmf: Logistic weighted profile-based collaborative matrix factorization for predicting mirna-disease associations. IEEE/ACM transactions on computational biology and bioinformatics (2019)

36. Ding $Y$, L.B. Lei X: Predicting mirna-disease associations based on multi-view variational graph auto-encoder with matrix factorization. IEEE journal of biomedical and health informatics (2021)

37. Liu, B., Zhu, X., Zhang, L., Liang, Z., Li, Z.: Combined embedding model for mirna-disease association prediction. BMC Bioinformatics 22(1) (2021)

38. Chen, X., Wang, L., Jia, Q., Guan, N.N., Li, J.Q.: Predicting mirna-disease association based on inductive matrix completion. Bioinformatics (24), 4256-4265 (2018)

39. Wang, D., Peng, C., Zhu, W.: Structural deep network embedding. In: Acm Sigkdd International Conference on Knowledge Discovery \& Data Mining (2016)

40. Grover, A., Leskovec, J.: node2vec: Scalable feature learning for networks. the 22nd ACM SIGKDD International Conference (2016)

41. Perozzi, B., Al-Rfou, R., Skiena, S.: Deepwalk: Online learning of social representations. ACM (2014)

42. Tang, J., Qu, M., Wang, M., Zhang, M., Yan, J., Mei, Q.: Line: Large-scale information network embedding. International Conference on World Wide Web Www (2015)

43. Hamilton, W.L., Ying, R., Leskovec, J.: Inductive representation learning on large graphs (2017)

44. Du, J., Zhang, S., Wu, G., Moura, J., Kar, S.: Topology adaptive graph convolutional networks (2017)

45. Wang, Ji, Kim., E.: micrornas in breast cancer: regulatory roles governing the hallmarks of cancer. Biological Reviews (2016)

46. Nicolson, S. Marianne: The impact of comorbidity upon determinants of outcome in patients with lung cancer. Lung cancer: Journal of the International Association for the Study of Lung Cancer 87(2), 186-192 (2015) 